\title{
Non-programmed gait generation of quadruped robot using pulse-type hardware neuron models
}

\author{
Yuki Takei $^{1} \cdot$ Katsuyuki Morishita $^{1} \cdot$ Riku Tazawa $^{1} \cdot$ Koichi Katsuya $^{1} \cdot$ Ken Saito $^{1}$
}

Received: 15 April 2020 / Accepted: 23 August 2020 / Published online: 14 September 2020

(c) The Author(s) 2020

\begin{abstract}
In this paper, the authors will propose the active gait generation of a quadruped robot. The theory that quadruped animals unconsciously generate gaits by some system based on neural networks in the spinal cord is widely accepted. However, how biological neurons or neural networks can generate gaits is not clear. To clarify the gait generation method, one of the solutions is using the neuron model similar to the biological neuron. We developed the quadruped robot system using selfinhibited pulse-type hardware neuron models (P-HNMs), which can output the electrical activity similar to those of biological neurons. The P-HNMs consist of the cell body model and the inhibitory synaptic model. The cell body model periodically outputs pulsed voltages; the inhibitory synaptic model inhibits the pulsed voltages. The pulse period can change by varying the synaptic weight control voltage applied to the P-HNMs. We varied the synaptic weight control voltage according to the pressure on the robot's toes. Also, we changed the angle of the robot's joints by a constant angle each time the P-HNMs output a pulse. As a result of the walking experiment, we confirmed that the robot generates walk gait and trot gait according to the moving speed. Also, we clarified the process by which the robot actively generates gaits from the upright state. These results show that animals may not use many biological neurons to generate gaits. Furthermore, the results suggest the possibility of realizing simple and bio-inspired robot control.
\end{abstract}

Keywords Gait generation · Pulse-type hardware neuron model $(\mathrm{P}-\mathrm{HNM}) \cdot$ Quadruped robot $\cdot$ Self-inhibit

\section{Introduction}

This work was presented in part at the 25th International Symposium on Artificial Life and Robotics (Beppu, Oita, January 22-24, 2020).

Yuki Takei

csyk19002@g.nihon-u.ac.jp

Katsuyuki Morishita

cska19034@g.nihon-u.ac.jp

Riku Tazawa

csri19021@g.nihon-u.ac.jp

Koichi Katsuya

csku20015@g.nihon-u.ac.jp

Ken Saito

kensaito@eme.cst.nihon-u.ac.jp

1 Nihon University, 7-24-1 Narashinodai, Funabashi, Chiba 274-8501, Japan
Quadruped animals change the gait according to the locomotion speed by adjusting leg movements [1]. The experiments using decerebrated cats show that gaits generated were unconsciously by some systems based on neural networks in the spinal cord [2-4]. Thus, implementing the gait generation mechanism of animals on a walking robot will allow the active gait generation with a simple control system. There are various studies for elucidating the mechanism by which animals gait generation method [5, 6]. However, the information processing of biological neurons is difficult to analyze. Therefore, research has been conducted to estimate the gait generation mechanism using robots.

Research using a biped machine with passive joints revealed that a gait pattern was generated without a control mechanism when the biped machine was placed on a shallow slope [7]. Also, research using a quadruped machine revealed that the gait of a quadruped animal was generated according to the type of body joints and the slope angle [8]. Furthermore, gaits can generate even if the number of legs 
increased to six or more [9]. These experiments suggest that gaits can be generated without a control mechanism even with machines. However, to realize a robot that can actively walk, it is necessary to study the gait generation mechanism including the driving method of the actuator.

Recently, a quadruped robot with joint using servomotors controlled by decoupled mathematical oscillating models based on the active rotator model has been proposed [10, 11]. The quadruped robot could generate an animal's gait according to the pressure on the quadruped's feet. Also, it has clarified that another robot controlled in the same method could switch the gaits according to its moving speed [12]. These results suggest the effectiveness of using the difference in pressure on each foot to generate gait. However, the oscillators used to control the joints were not designed on a biological basis. The authors think that spike firing has a significant role in information processing in the brain.

The authors are studying robot control using pulse-type hardware neuron models (P-HNMs). The P-HNMs can output the spike firing (action potential) similar to a biological neuron [13]. Our research aims to develop a simple and efficient control method for robots using the artificial motor nervous system and central nervous systems. Therefore, hardware implementation will be advantageous in a largescale network system.

Previously, we have developed a quadruped robot system that implemented a gait generation mechanism using P-HNMs. The mechanism is similar to the peripheral nervous system in that independent P-HNMs control each limb individually. Also, we revealed that the robot system generates two kinds of gaits depending on the moving speed [14]. However, the process by which gaits generated is not clear. In this paper, the authors improved the quadruped robot to analyze how the gaits generated by the method we proposed. First, we will introduce the components of our quadruped robot system. Second, we will discuss the gait generation method, and finally, we will show the experimental result.

\section{Quadruped robot system}

Figure 1 shows our constructed quadruped robot system. This chapter describes the individual components of the quadruped robot system.

\subsection{Mechanical and electrical components}

The quadruped robot consists of mechanical and electrical components. The mechanical components of the robot system consist of the body frame and four legs. The body frame was made from an aluminum alloy (A5052). Some parts of the legs were made from polylactic acid (PLA). The robot's leg length is $138 \mathrm{~mm}$, the distance between the front and rear

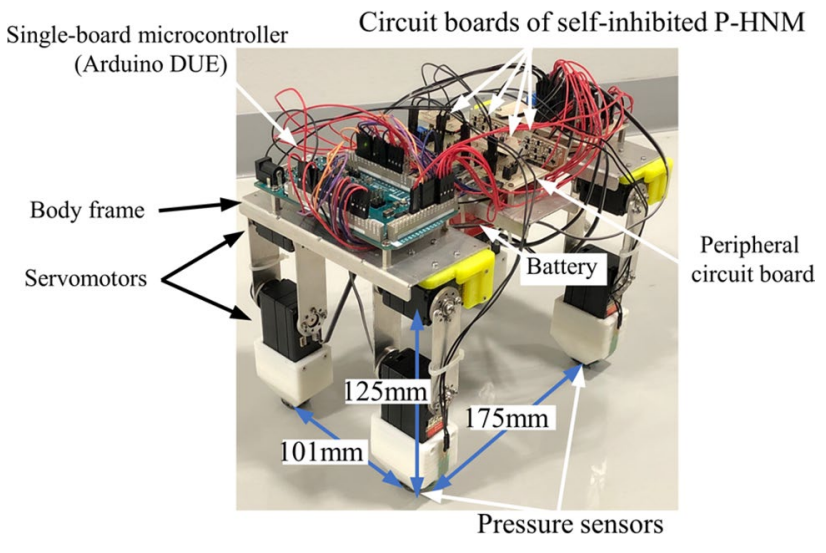

Fig. 1 Constructed quadruped robot system

legs is $170 \mathrm{~mm}$, and the distance between the left and right legs is $101 \mathrm{~mm}$. The total weight of the robot is approximately $1.1 \mathrm{~kg}$. This robot has no degrees of freedom except for legs to focus on the gait generation using the pressure underside of the foot. All the legs have the same structure, where the legs have two joints on the same axis using servomotors (Kondo Kagaku: KRS-2552).

The electrical components of the robot system consist of circuit boards of self-inhibited P-HNM, pressure sensors (Interlink Electronics: FSR402), single-board microcontroller (Arduino DUE), and peripheral circuit board. The pressure sensors have attached to the feet. Also, we mounted a battery and Bluetooth module. Therefore, we were able to proceed with the walking experiment of the robot system without physical connections for power supply and communication.

\subsection{Leg controlling system}

We set two commands in the microcontroller to move the legs. One is to read the output of the pressure sensor and output the voltage accordingly. The other is to change the angle of the servomotors by a constant angle each time the voltage input to the interrupt pin exceeds $1.7 \mathrm{~V}$ (microcontroller's interruption voltage). When changing the angle of servomotors, the foot passes through the four target points and moves along the trajectory shown in Fig. 2. We set the microcontroller to process these commands individually for four legs.

\subsection{Self-inhibited P-HNM}

Figure 3 shows the schematic and circuit diagram of the self-inhibited P-HNM that consists of a cell body model and an inhibitory synaptic model. The schematic diagram at the top of Fig. 3 shows the connection between the cell body model and the inhibitory neuron model, with a large 


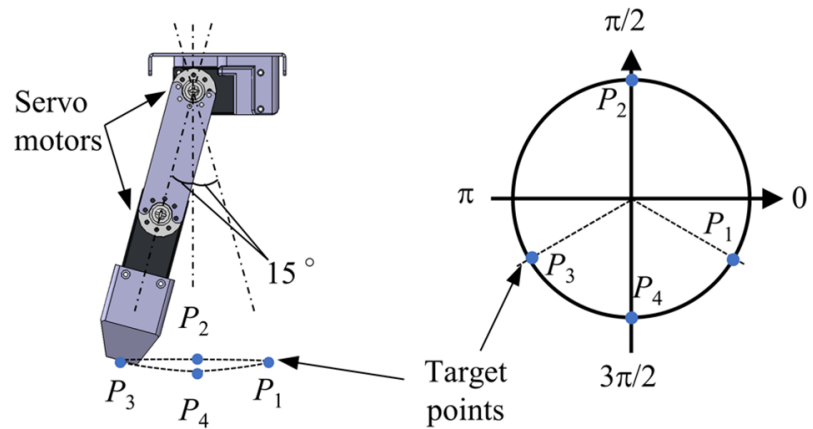

(a) Schematic diagram

(b) Phases of target points

Fig. 2 Foot trajectory

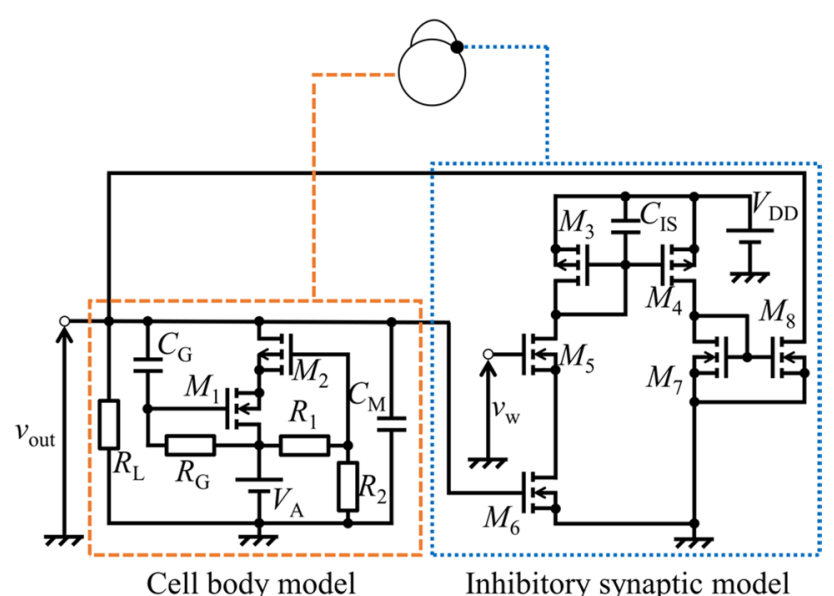

Fig. 3 Schematic and circuit diagram of self-inhibited P-HNM

circle representing the cell body model and black circle and arc representing the inhibitory neuron model. The cell body model and the inhibitory synaptic model are mimicking several functions of a biological neuron. The cell body model includes a voltage control-type negative resistance, an equivalent inductance, resistors $R_{1}$ and $R_{2}$, and a membrane capacitor $C_{\mathrm{M}}$. The voltage control-type negative resistance circuit with equivalent inductance consists of an n-channel MOSFET $M_{1}$, a p-channel MOSFET $M_{2}$, a voltage source $V_{\mathrm{A}}$, a leak resistor $R_{\mathrm{L}}$, another resistor $R_{\mathrm{G}}$, and a capacitor $C_{\mathrm{G}}$. The cell body model generates oscillating patterns of electrical activity $v_{\text {out }}$. More detail of the cell body model is described in [13]. The inhibitory synaptic model consists of simple current mirror circuits. The function of the inhibitory synaptic model is to inhibit the generation of the pulses by the cell body model. The inhibitory synaptic model inhibits pulse generation by pulling out current from the cell body model. The strength of the inhibition can vary with synaptic weight control voltage $v_{\mathrm{w}}$.

Figure 4 shows the simulation result of waveform output by self-inhibited P-HNM. We changed $v_{\mathrm{w}}$ applied to

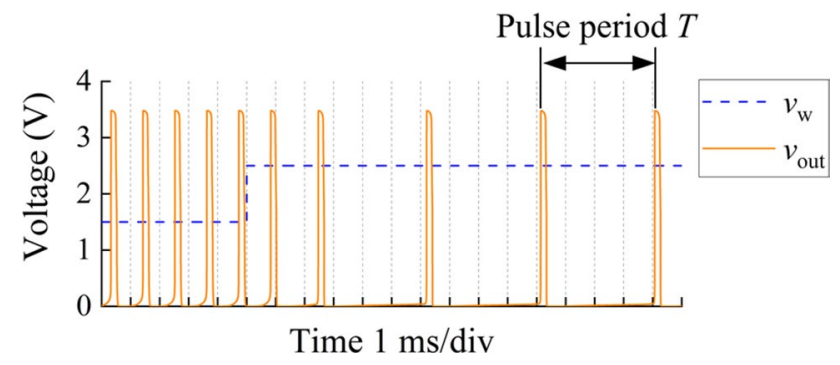

Fig. 4 Example of output pulse waveform (simulation result)

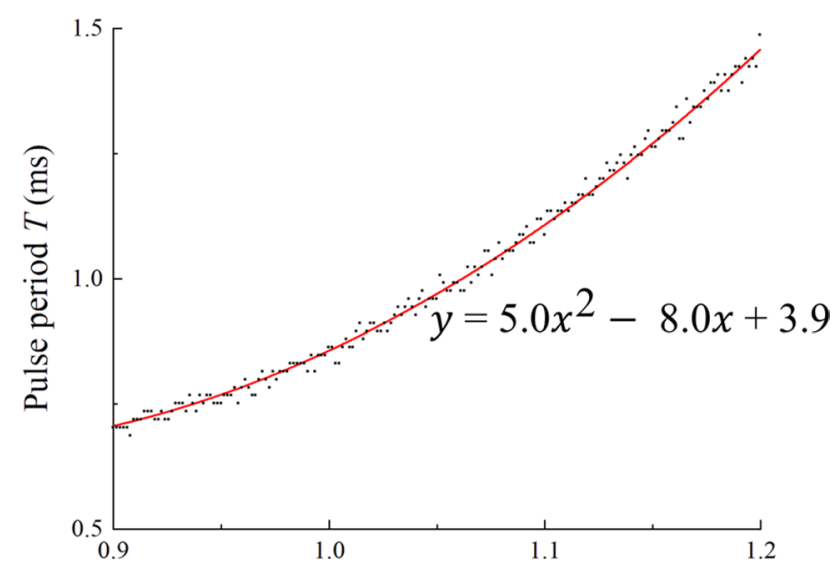

Synaptic weight control voltage $v_{\mathrm{w}}(\mathrm{V})$

Fig. 5 Pulse period characteristics of self-inhibited P-HNM (experimental result)

the self-inhibited P-HNM in the middle of this simulation. The circuit constants are $C_{\mathrm{IS}}=3.3 \mu \mathrm{F}, C_{\mathrm{G}}=47 \mathrm{pF}, C_{\mathrm{M}}=10$ $\mathrm{pF}, R_{1}=15 \mathrm{k} \Omega, R_{2}=20 \mathrm{k} \Omega, R_{\mathrm{G}}=8.2 \mathrm{M} \Omega, R_{\mathrm{L}}=10 \mathrm{k} \Omega$, $M_{1,5,6,7,8}: \mathrm{BSS} 83, M_{2,3,4}: \mathrm{BSH} 205, V_{\mathrm{DD}}=5.0 \mathrm{~V}, V_{\mathrm{A}}=3.5 \mathrm{~V}$.

Figure 5 shows the result of the measured relation between the pulse period $T$ and the synaptic weight control voltage $v_{\mathrm{w}}$ of the self-inhibited P-HNM. The curve in Fig. 5 is the result of approximating the plotted points with a second-order polynomial, the region we used to control the robot.

\subsection{Connection of components}

We connected these components to realize the quadruped robot system with active gait generating function. Figure 6 shows the connection of the single-leg components and circuit diagram of the peripheral circuit. The peripheral circuit includes a low-pass filter, buffer and voltage dividing circuits. The low-pass filter consists of a resistor $R_{\mathrm{F}}$ and a capacitor $C_{\mathrm{F}}$. The buffers consist of operational amplifier $U_{1}$ and $U_{2}$. The voltage dividing circuits consist of $R_{\mathrm{D} 1}$, 


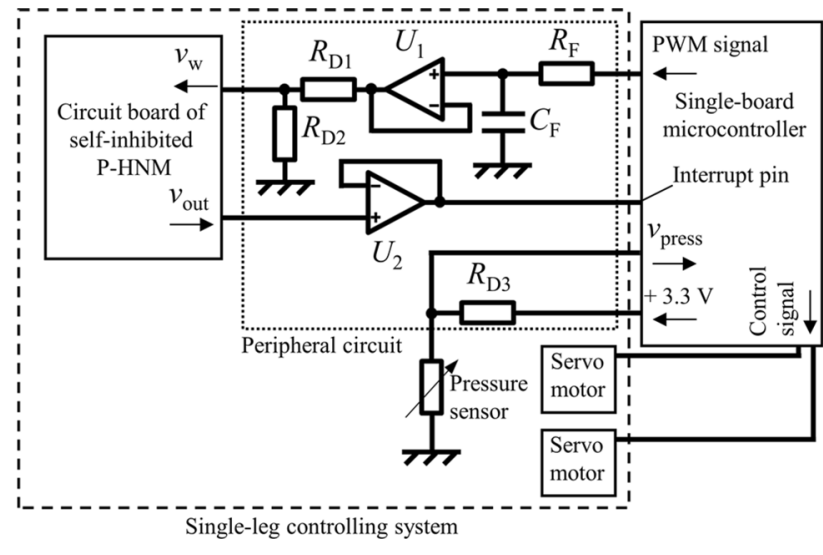

Fig. 6 Connection of single-leg controlling system and circuit diagram of peripheral circuit

$R_{\mathrm{D} 2}$, and $R_{\mathrm{D} 3}$. The circuit constants of peripheral circuit are $C_{\mathrm{F}}=3.3 \mu \mathrm{F}, R_{\mathrm{F}}=11 \mathrm{k} \Omega, R_{\mathrm{D} 1, \mathrm{D} 2, \mathrm{D} 3}=11 \mathrm{k} \Omega, U_{1,2}: \mathrm{LMC} 6032$.

We input the PWM output of the microcontroller to the circuit board of self-inhibited P-HNM as an analog output through the low-pass filter of the peripheral circuits. The voltage output by the circuit board of self-inhibited P-HNM applied to the microcontroller's interrupt pin through the peripheral circuit. Thus, we allowed the robot system to change the speed at which each leg moves in real time. Each leg moves by a constant angle each time the circuit boards of self-inhibited P-HNM output a pulse, and the period at which the P-HNMs output a pulse varies depending on the pressure. Therefore, the speed at which each leg of the robot moves varies depending on the pressure on the feet.

\section{Gait generation method}

The following equations express the relation between the speed of moving legs and the pressure on the feet. The microcontroller controlled the legs individually. Therefore, some parameters are different for each leg. In the following equations, the subscript " $i$ " means the parameter for the $i$ th leg. The angular velocity of moving legs $\omega_{i}$ can be described as the following equation:

$\omega_{i}=\frac{\theta}{T_{i}}$,

where $\theta$ is an actuation angle of servomotors each time the circuit board of self-inhibited P-HNM output a pulse. The synaptic weight control voltage $v_{\mathrm{w}}$ applied to the circuit board of self-inhibited P-HNM can be described as the following equation:

$v_{\mathrm{w} i}=\sigma v_{\text {pressi }}$,

where $v_{\text {pressi }}$ is the applied voltage to the microcontroller depending on output by the pressure sensors. $\sigma$ is a constant for converting $v_{\text {pressi }}$ to $v_{\mathrm{w} i}$, and represents the effect of pressure. From the approximate formula in Fig. 5, the pulse period $T_{i}$ of the output voltage of self-inhibited P-HNMs $v_{\text {out }}$ can be described as the following equation:

$T_{i}=5.0 v_{\mathrm{w} i}^{2}-8.0 v_{\mathrm{w} i}+3.9$.

From these equations, $\omega_{i}$ can describe as the following equation. This equation indicates that the pressure on the foot reduces the angular velocity of moving the leg.

$\omega_{i}=\frac{\theta}{5.0 v_{\mathrm{w} i}^{2}-8.0 v_{\mathrm{w} i}+3.9}$.

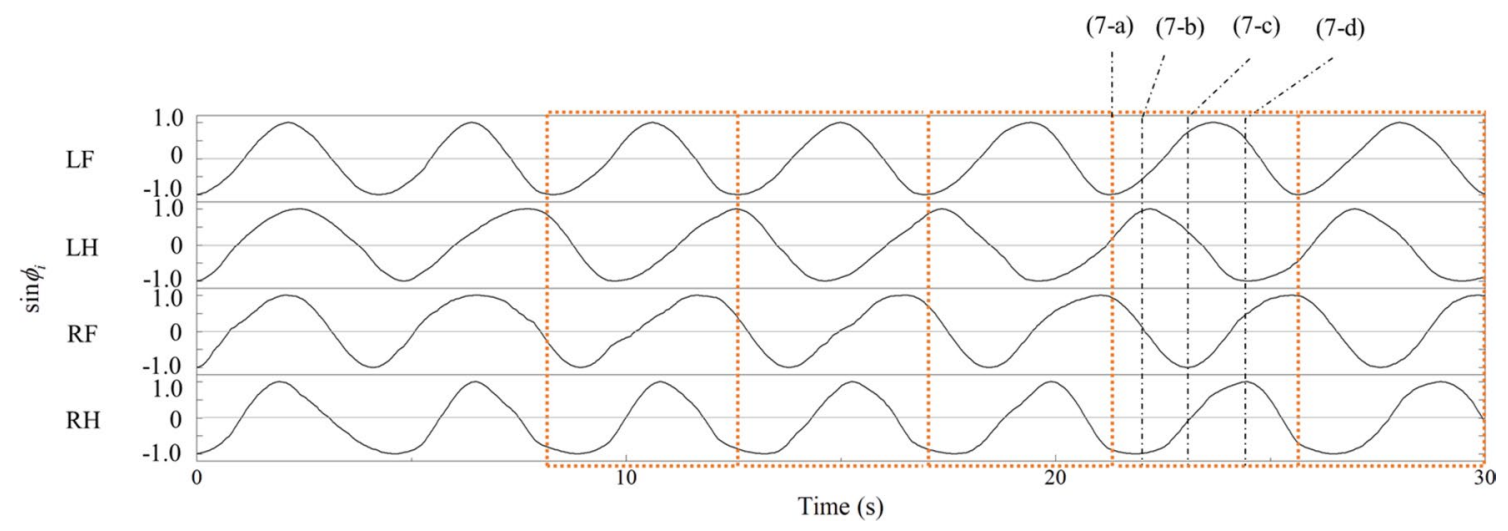

Fig. 7 Phase transition of legs at low speed (walk gait) 


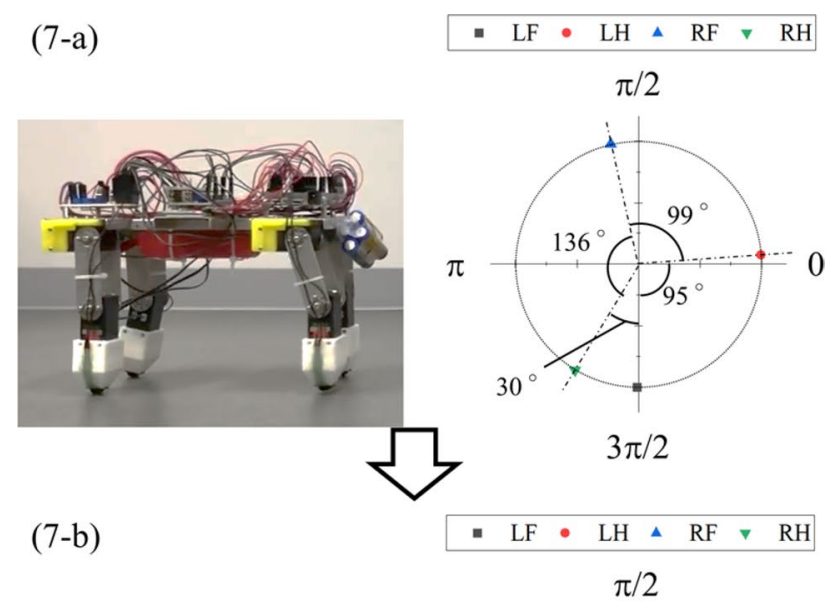

a flat floor. We conducted experiments under two conditions: when the robot's walking speed is slow and when it is fast. To change the robot's walking speed, we changed the angular velocity of the legs by changing $\theta$. However, we did not change parameters such as $\sigma$. We set the initial phase of each leg to $3 \pi / 2$ and let the legs to start moving at the same time.

Figures 7 and 8 show the transition of each leg's phase $\phi_{i}$ and the generated phase difference at low speed. The numbers in Fig. 8 correspond to the times indicated by numbers in Fig. 7. The borders in Fig. 7 mean one cycle of gait. As Figs. 7 and 8 show from the third step after the robot starts walking, the phase difference of each leg was kept around $90^{\circ}$. Also, the order of moving the legs is left fore (LF), right hind $(\mathrm{RH})$, right fore (RF), and left hind (LH), which means that this gait is the same as the horse's walk gait. In this experiment, the angular velocity of the legs while the legs were not on the floor was approximately $30 \% \mathrm{~s}(0.52 \mathrm{rad} / \mathrm{s})$.

Figures 9 and 10 show the result at high speed. As Figs. 9 and 10 show from the fourth step after the robot starts walking, the phase difference of each leg was kept around $180^{\circ}$. In addition, the order of moving the legs is LF and RH, RF and LH, which means that this gait is the same as the horse's trot gait. In this experiment, the angular velocity of the legs while the legs were not on the floor was approximately $51 \%$ (0.89 rad/s). These results show that the quadruped robot system can generate gaits by reducing the angular velocity of the legs depending on the pressure on the feet. Also, the robot system can generate different gaits depending on moving speed. Furthermore, the characteristics of the generated gaits are similar to the horse's gaits. In our control method, we confined the factor of change in each leg's speed to feedback using weight-bearing balance. Therefore, we assume that the trigger for the break in the initial phase symmetry was slightly different in the weight of the robot that the limbs were supporting.

We have experimentally determined the parameters such as $\theta$ and $\sigma$ that can stably produce these gestures. We expect that the dynamics simulator is necessary to determine these parameters quantitatively. In the future, we will use it to analyze in detail how the parameters affect the gaits.

\section{Conclusion}

In this paper, the authors constructed a quadruped robot controlled by the active gait generating method individually for four legs. The method is simply reducing the moving speed of the robot's legs according to the pressures of feet using pulse-type hardware neuron models (P-HNMs). We conducted experiments under two conditions: when the robot's walking speed is slow and when it is fast. As a result, the robot system actively generated phase differences of each leg. By analyzing the experimental results, we clarified the

To analyze the gaits generated by the robot system and the process of generating gaits, we let the robot system walk on 
Fig. 9 Phase transition of legs at high speed (trot gait)

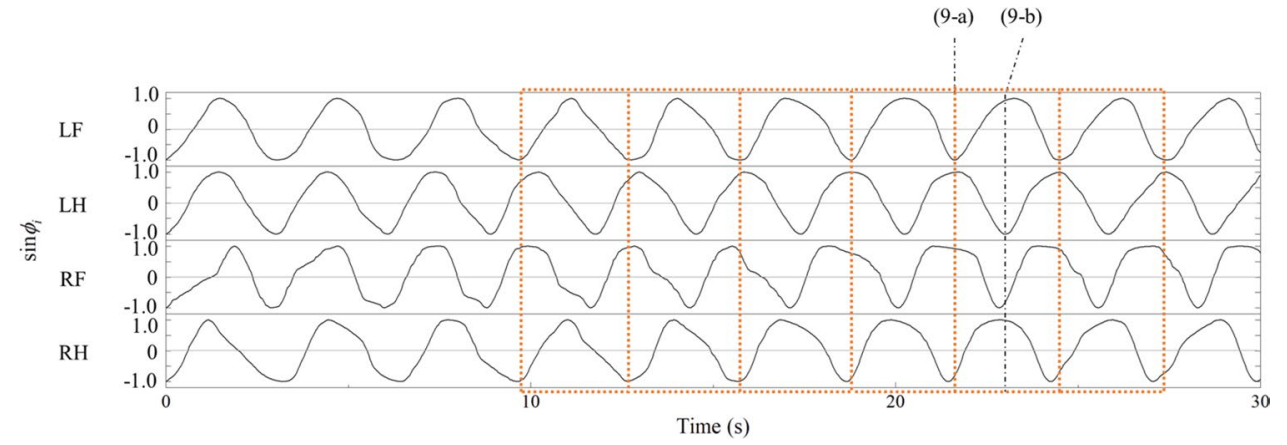

(9-a)

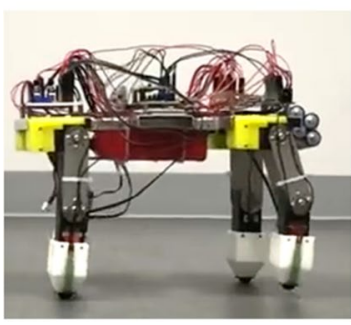

- $\mathrm{LF} \bullet \mathrm{LH} \wedge \mathrm{RF} \vee \mathrm{RH}$ $\pi / 2$

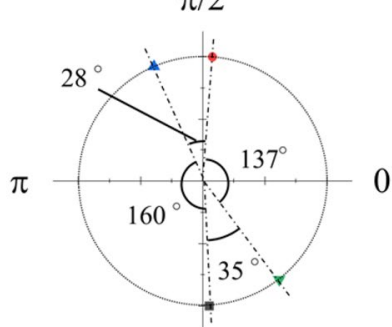

$3 \pi / 2$ $\sqrt{5}$

(9-b)

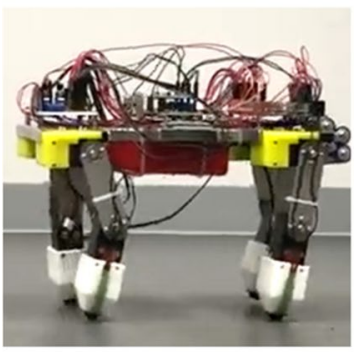

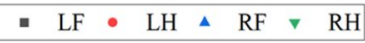

$\pi / 2$

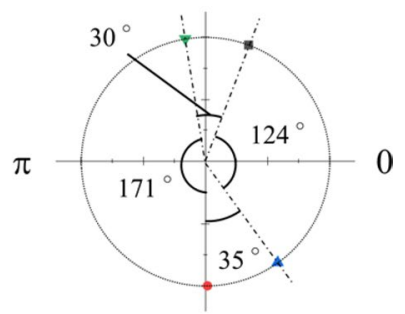

$3 \pi / 2$

Fig. 10 Quadruped robot system and generated phase difference at high speed (9-a, 9-b, 9-c, and 9-d indicate the time points of Fig. 9)

process of gait generation. Also, we confirmed that the generated phase differences were similar to the horse's gaits of walk and trot. These results show that animals may not use many biological neurons to generate gaits. Furthermore, the results suggest the possibility of realizing simple and bioinspired robot control.

Acknowledgements This work was supported by JSPS KAKENHI Grant Number JP18K04060 and supported by Research Institute of Science and Technology Nihon University Collage of Science and Technology Leading Research Promotion Grant. Also, we appreciated to the Nihon University Robotics Society (NUROS).
Open Access This article is licensed under a Creative Commons Attribution 4.0 International License, which permits use, sharing, adaptation, distribution and reproduction in any medium or format, as long as you give appropriate credit to the original author(s) and the source, provide a link to the Creative Commons licence, and indicate if changes were made. The images or other third party material in this article are included in the article's Creative Commons licence, unless indicated otherwise in a credit line to the material. If material is not included in the article's Creative Commons licence and your intended use is not permitted by statutory regulation or exceeds the permitted use, you will need to obtain permission directly from the copyright holder. To view a copy of this licence, visit http://creativecommons.org/licenses/by/4.0/.

\section{References}

1. Hoyt DF, Taylor CR (1981) Gait and the energetics of locomotion in horses. Nature 292:239-240

2. Brown TG, Sherrington CS (1911) The intrinsic factors in the act of progression in the mammal. Proc R Soc B 84(572):308-319

3. Gorassini MA, Prochazka A, Hiebert G, Gauthier MA (1994) Corrective responses to loss of ground support during walking I. Intact cats. J Neurophysiol 71(2):603-610

4. Hiebert GW, Gorassini MA, Jiang W, Prochazka A (1994) Corrective responses to loss of ground support during walking II Comparison of intact and chronic spinal cats. J Neurophysiol 71(2):611-621

5. Grillner S (1985) Neurobiological bases of rhythmic motor acts in vertebrates. Science 228(4696):143-149

6. Collins J, Stewart N (1993) Coupled nonlinear oscillators and the symmetries of animal gaits. J Nonlinear Sci 3(1):349-392

7. McGeer T (1990) Passive dynamic walking. Int J Robot Res 9(2):62-82

8. Nakatani K, Sugimoto Y, Osuka K (2009) Demonstration and analysis of quadrupedal passive dynamic walking. Adv Robot 23(5):483-501

9. Sugimoto Y, Yoshioka H, Osuka K (2010) The realization of the super multilegged passive dynamic walking (in Japanese). J Robot Soc Jpn 28(8):961-969

10. Shinomoto S, Kuramoto Y (1986) Phase transitions in active rotator systems. Progress Theor Phys 75(5):1105-1110

11. Owaki D, Kano T, Nagasawa K, Tero A et al (2013) Simple robot suggests physical interlimb communication is essential for quadruped walking. J R Soc Interface 10(78):20120669

12. Owaki D, Ishiguro A (2017) A quadruped robot exhibiting spontaneous gait transitions from walking to trotting to galloping. Sci Rep 7(277):1-10

13. Saito K, Ohara M, Abe M, Kaneko M et al (2018) Gait generation of multilegged robots by using hardware neural networks. 
In: El-Shahat A (ed) Advanced applications for artificial neural networks. IntechOpen, Rijeka, pp 34-36

14. Takei Y, Morishita K, Tazawa R, Kaneko M et al (2019) Development of quadruped robot generating animal-like gaits utilizing independent neuro-circuits (in Japanese). In: Proceedings of the 32nd workshop on circuits and systems, pp 222-227
Publisher's Note Springer Nature remains neutral with regard to jurisdictional claims in published maps and institutional affiliations. 